\title{
Secret Sharing and Non-Shannon Information Inequalities *
}

\author{
Amos Beimel and Ilan Orlov \\ Dept. of Computer Science, Ben-Gurion University Be'er-Sheva, Israel
}

\begin{abstract}
The known secret-sharing schemes for most access structures are not efficient; even for a one-bit secret the length of the shares in the schemes is $2^{O(n)}$, where $n$ is the number of participants in the access structure. It is a long standing open problem to improve these schemes or prove that they cannot be improved. The best known lower bound is by Csirmaz (J. Cryptology 97), who proved that there exist access structures with $n$ participants such that the size of the share of at least one party is $n / \log n$ times the secret size. Csirmaz's proof uses Shannon information inequalities, which were the only information inequalities known when Csirmaz published his result. On the negative side, Csirmaz proved that by only using Shannon information inequalities one cannot prove a lower bound of $\omega(n)$ on the share size. In the last decade, a sequence of nonShannon information inequalities were discovered. This raises the hope that these inequalities can help in improving the lower bounds beyond $n$. However, in this paper we show that all the inequalities known to date cannot prove a lower bound of $\omega(n)$ on the share size.
\end{abstract}

\section{Introduction}

A secret-sharing scheme is a mechanism for sharing data among a set of participants such that only pre-defined authorized subsets of participants can reconstruct the data, while any other subset has absolutely no information on the data. The collection of authorized subsets is called an access structure. For example, in a $t$-out-of- $n$ threshold secret-sharing scheme, the access structure contains all subsets of size at least $t$. As an interesting "real-world" illustration of this situation: According to Time Magazine control of the nuclear weapon in Russia in the early 1990s depended upon a similar "two-out-of-tree" access mechanism, where the three parties were the President, the Defense Minister, and the Defense Ministry. Secret-sharing schemes, introduced by 40, 8, 30, are nowadays used in many cryptographic protocols, e.g., Byzantine agreement [38, secure multiparty computations [6, 14, 17, threshold cryptography [20, access control 36], and attribute-based encryption [27, 43].

An important issue in secret-sharing schemes is the size of the shares distributed to the participants. For most access structures, even the best known

The original version of this chapter was revised: The copyright line was incorrect. This has been corrected. The Erratum to this chapter is available at DOI: 10.1007/978-3-642-00457-5_36

* Partially supported by the Frankel Center for Computer Science at the Ben-Gurion University. 
secret-sharing schemes (e.g., 7, 11, 21, 31, 42, 31) are not efficient; the length of the shares for sharing an $\ell$-bit secret is $\ell \cdot 2^{O(n)}$, where $n$ is the number of participants in the access structure. The best lower bound was proved by Csirmaz 18; he proved that for each $n$ there exists an access structure with $n$ participants such that any secret-sharing scheme with an $\ell$-bit secret requires shares of length $\Omega(\ell n / \log n)$. There is a large gap between the upper bounds and the lower bounds. Closing this gap is a major open problem.

The entropy of a random variable, which was introduced by Shannon in the landmark paper 41], is a measure of the amount of uncertainty associated with the value of the random variable. Starting from the works of Karnin et al. [32. and Capocelli et al. 12, the entropy was used to prove lower bounds on the share size in secret sharing schemes [9, 22, 18, 19. Specifically, Csirmaz's proof [1] uses only Shannon information inequalities, which were the only information inequalities known when Csirmaz published his result (this is true also for all the previous works mentioned above). On the negative side, Csirmaz proved that by using only Shannon information inequalities one cannot prove a lower bound of $\omega(n)$ on the share size. In the last decade, a sequence of non-Shannon information inequalities were discovered. This raises the hope that these inequalities can help in improving the lower bounds beyond $n$. However, in this paper we show that all the inequalities known to date cannot prove a lower bound of $\omega(n)$ on the share size.

\section{$1.1 \quad$ Related Work}

Threshold secret-sharing schemes, in which a subset is authorized iff its size is larger than some threshold, were independently introduced by Shamir 40 and Blakley [8] about thirty years ago. General secret sharing schemes were presented by Ito, Saito, and Nishizeki 30] they presented a construction of a secret-sharing scheme for every monotone access structure. More efficient schemes for specific access structures were presented in, e.g., 7, 11, 21, 42, 31]. However, even these better constructions are not efficient and, for most access structure, the shares' size is exponential. Lower bounds for secret-sharing schemes were presented in 9 , 22. 18. 19]; however, as stated above, there is a big gap between the upper and lower bounds. Super-polynomial lower bounds for linear secret-sharing schemes were presented in [1, 26.

In this work, we discuss using information inequalities for proving lower bounds on the share size in secret-sharing schemes. An information inequality is a linear inequality over the entropy of subsets of variables that holds for any random variables (for a formal definition see Section [2.1). For example, $H\left(X_{1}\right)+H\left(X_{2}\right) \geq H\left(X_{1} X_{2}\right)$ is an information inequality. Many inequalities can be expressed as a linear combination of a single inequality involving the conditional mutual information, namely, $I(X ; Y \mid Z) \geq 0$. Such inequalities are known as Shannon inequalities. It was an open problem for many years if there are information inequalities that are not implied by Shannon inequalities, i.e., if there are non-Shannon inequalities. The first non-Shannon inequality was given by Zhang and Yeung [4]. In the last decade, several additional non-Shannon 
inequalities were discovered [33, 46, 23, 44. In particular, an interesting technique for deriving non-Shannon inequalities, called projection, was presented in 44. Several papers have dealt with the characterization of information inequalities. Chan and Yeung [13. have characterized information inequalities using group-theoretic inequalities. Matúš 34 has proved that there are infinitely many independent information inequalities. Guille et al. 28 have given results concerning the structure of information inequalities and, more specially, results describing the minimal set of information inequalities when all the coefficient are 1 or -1 , called Ingleton inequalities.

The information inequality of Zhang and Yeung 47. was used in several areas. It was used by Dougherty, Freiling, and Zeger [24] to prove bounds on the capacity of network coding, by Matús 35] to prove that a function is not asymptotically entropic, and by Riis 39] to prove bounds on graph entropy of certain graphs. Furthermore, it was used by Beimel, Livne, and Padró [4] to prove lower bounds on the size of shares in secret-sharing schemes; they proved that there is a matroidial access structure - the Vamos access structure - that is not nearly ideal. We observe that this result can be proved using other information inequalities, e.g., the information inequalities of 23. Furthermore, the information inequalities of 47, 23 can be used to prove that other matroidial access structures are not nearly ideal, e.g., the access structures induced by the matroids AG32r, F8, Q8 (for the definitions of these matroids see [37]).

This paper deals with limitations of the techniques for proving lower bounds on the size of shares in secret-sharing schemes, continuing the work of [3]. Beimel and Franklin [3] considered weakly-private secret-sharing schemes, in which any unauthorized set can never rule-out any secret (however, it might deduce, for example, that one secret is much less likely than other secrets). They show efficient constructions of weakly-private secret-sharing schemes (for large secret domains), implying that to prove lower bounds on the shares' size in secretsharing schemes one must use the strong privacy requirement of secret-sharing schemes.

\subsection{Our Results}

In contrast to the success of applying the known information inequalities to proving lower bounds in several areas, we show that they cannot help in proving lower bounds of $\omega(n)$ on the share size in secret-sharing schemes. Let us elaborate on our proof. Csirmaz [18] in 1994 has proven his lower bound by translating the question of proving lower bounds on share size to proving that a certain linear programming instance does not have a small solution. Csirmaz constructed the linear program by using Shannon inequalities, which were the only information inequalities known in 1994. He proved a lower bound of $\Omega(n / \log n)$ times the secret size for an access structure with $n$ parties. Furthermore, all previous lower bounds 32, 12, 9, 22 can be restated using Csirmaz's framework using Shannon inequalities. On the other hand, Csirmaz proved that for every access structure the linear program has a solution in which the objective function has value $O(n)$, implying that his framework cannot prove better lower bounds than $\Omega(n)$. 
In the last decade, a sequence of non-Shannon information inequalities were discovered [47, 33, 46, 23, 44. This gives hope that adding these inequalities to the linear program, one could prove better lower bounds on the share size. However, in this work we show that Csirmaz's solution to the linear program remains valid even after adding all the known information inequalities. That is, all the information inequalities known to date cannot prove lower bounds better than $\Omega(n)$ even if used simultaneously. Our proof that Csirmaz's solution remains valid after adding the new inequalities is much more involved than Csirmaz's proof for Shannon inequalities. We present a brute-force algorithm that checks if Csirmaz's solution remains valid given an information inequality 1 We executed this algorithm, using a computer program, on all known information inequalities of 47, 33, 46, 23. For 47, 46, 33, 44, which also give an infinite sequence of information inequalities, we manually executed the algorithm on a symbolic representation of the inequalities. The conclusion is that all the known information inequalities cannot help in proving better lower bounds than $\Omega(n)$.

We end the introduction with a few remarks. First, one cannot interpret our result as suggesting that information inequalities cannot help in improving the lower bounds. To the contrary, the conclusion of our paper is that new information inequalities should be sought. Hopefully, these new information inequalities would not be ruled-out by our algorithm. However, not failing the test in our algorithm is only the first step. Our algorithm only gives a necessary condition for an information inequality to be helpful in proving lower bounds of $\omega(n)$ on the share size. To use new inequalities, one has to prove that for some access structure the linear program with the new inequalities, and possibly with all the known inequalities, has only large solutions.

\section{Preliminaries}

In this section we review the relevant definitions from information theory and define secret-sharing schemes.

\subsection{Basic Definitions from Information Theory and Information Inequalities}

In this section, we review the basic concepts of Information Theory used in this paper. For a complete treatment of this subject see, e.g., [16]. All the logarithms here are of base 2 .

The entropy of a random variable $X$ is $H(X) \stackrel{\text { def }}{=}-\sum_{x, \operatorname{Pr}[X=x]>0} \operatorname{Pr}[X=$ $x] \log \operatorname{Pr}[X=x]$. It can be proved that $0 \leq H(X) \leq \log |\operatorname{supp}(X)|$, where $|\operatorname{supp}(X)|$ is the size of the support of $X$ (the number of values with probability greater than zero). The upper bound $|\operatorname{supp}(X)|$ is obtained if and only if the distribution of $X$ is uniform and the lower bound is obtained if and only if $X$ is

\footnotetext{
${ }^{1}$ Our algorithm is highly inefficient. However, most known non-Shannon information inequalities have 4 or 5 variables, thus, executing the computer program returns an answer in a reasonable time (less than a minute).
} 
deterministic. Given two random variables $X$ and $Y$ (possibly dependent), the conditioned entropy of $X$ given $Y$ is defined as $H(X \mid Y) \stackrel{\text { def }}{=} H(X, Y)-H(Y)$. From the definition of the conditional entropy, the following properties can be proved: $0 \leq H(X \mid Y) \leq H(X)$, where $H(X \mid Y)=H(X)$ if and only if $X$ and $Y$ are independent, and $H(X \mid Y)=0$ if the value of $Y$ completely determines the value of $X$. The mutual information between $X$ and $Y$ is defined as $I(X ; Y) \stackrel{\text { def }}{=}$ $H(X)-H(X \mid Y)$, and the conditional mutual information between $X$ and $Y$ given $Z$ is defined as $I(X ; Y \mid Z) \stackrel{\text { def }}{=} H(X \mid Z)-H(X \mid Y, Z)$. Entropies, conditional entropies, mutual information, and conditional mutual information are called Shannon's information measures.

Let $\left\{X_{i}\right\}_{i \in[m]}$ be a set of $m$ jointly distributed random variables. For any subset $I$ of $[m]$, let $X_{I}=\left(X_{i}\right)_{i \in I}$.

Definition 1 (Information Inequality). An information inequality over $m$ variables is defined by $2^{m}$ constants $\left\{\alpha_{A}\right\}_{A \subseteq[m]}$, where $\alpha_{A} \in \mathbb{R}$, such that $\sum_{A \subseteq[m]} \alpha_{A} H\left(X_{A}\right) \geq 0$ for every $m$ random variables $X_{1}, \ldots, X_{m}$.

For example, $H\left(X_{1}\right)+H\left(X_{2}\right) \geq H\left(X_{1} X_{2}\right)$ is an information inequality. Many inequalities can be expressed as a linear combination of a single inequality involving the conditional mutual information, namely, $I\left(X_{1} ; X_{2} \mid X_{3}\right) \geq 0$ (this inequality can be stated as $\left.H\left(X_{1}, X_{3}\right)+H\left(X_{2}, X_{3}\right)-H\left(X_{1}, X_{2}, X_{3}\right)-H\left(X_{3}\right) \geq 0\right)$. Such inequalities are known as Shannon-type inequalities. Information inequalities that cannot be deduced from Shannon inequalities are called non-Shannon inequalities. For more background on information inequalities the reader may consult [45].

\section{$2.2 \quad$ Secret Sharing}

Definition 2 (Access Structure and Distribution Scheme). Let $P=$ $\left\{p_{1}, \ldots, p_{n}\right\}$ be a finite set of parties, and let $p_{0} \notin P$ be a special party called the dealer. $A$ collection $\mathcal{A} \subseteq 2^{P}$ is monotone if $B \in \mathcal{A}$ and $B \subseteq C$ imply that $C \in \mathcal{A}$. An access structure is a monotone collection $\mathcal{A} \subseteq 2^{P}$ of non-empty subsets of $P$. Sets in $\mathcal{A}$ are called authorized, and sets not in $\mathcal{A}$ are called unauthorized.

$A$ distribution scheme $\Sigma=\langle\Pi, \mu\rangle$ with domain of secrets $K$ is a pair, where $\mu$ is a probability distribution on some finite set $R$ (the set of random strings) and $\Pi$ is a mapping from $K \times R$ to a set of $n$-tuples $K_{1} \times K_{2} \times \cdots \times K_{n}$, where $K_{i}$ is called the share-domain of $p_{i}$. A dealer distributes a secret $s \in K$ according to $\Sigma$ by first sampling a string $r \in R$ according to $\mu$, computing a vector of shares $\Pi(s, r)=\left(s_{1}, \ldots, s_{n}\right)$, and privately communicating each share $s_{i}$ to party $p_{i}$.

We next define secret-sharing schemes using the entropy function. It is convenient to view the secret as the share of the dealer $p_{0}$, and for every set $T \subseteq P \cup\left\{p_{0}\right\}$ to consider the vector of shares of $T$. Any probability distribution on the domain of secrets, together with the distribution scheme $\Sigma$, induces, for any $T \subseteq P \cup\left\{p_{0}\right\}$, a probability distribution on the vector of shares of the parties in $T$. We denote the random variable taking values according to this probability distribution on 
the vector of shares of $T$ by $S_{T}$, and by $S$ the random variable denoting the secret (i.e., $S=S_{\left\{p_{0}\right\}}$ ).

Definition 3 (Secret-Sharing Scheme). We say that a distribution scheme is a secret-sharing scheme realizing an access structure $\mathcal{A}$ with respect to a given probability distribution on the secrets, denoted by a random variable $S$, if the following conditions hold.

Correctness. For every authorized set $T \in \mathcal{A}$, the shares of the parties in $T$ determine the secret, i.e., $H\left(S \mid S_{T}\right)=0$.

PrIVACY. For every unauthorized set $T \notin \mathcal{A}$, the shares of the parties in $T$ do not disclose any information on the secret, that is, $H\left(S \mid S_{T}\right)=H(S)$.

Remark 1. Although the above definition considers a specific distribution on the secrets, Blundo et al. [10] proved that its correctness and privacy are actually independent of this distribution: If a scheme realizes an access structure with respect to one distribution on the secrets, then it realizes the access structure with respect to any distribution with the same support. Furthermore, the above definition is equivalent to the definition of [15, 2, 15, where there is no probability distribution associated with the secrets and it is required that the probability of every vector of shares of an unauthorized set is the same given any secret.

Karnin et al. 32] have showed that for each non-redundant party (that is, a party that appears in at least one minimal authorized set) $H\left(S_{i}\right) \geq H(S)$, which implies that the size of the share of the party is at least the size of the secret.

Notation 1. We use the following notation for two sets $A$ and $\widehat{A}$. The set $\widehat{A}$ is a subset of $P \cup\left\{p_{0}\right\}$ and the set $A$ is a subset of $P$, where $A=\widehat{A} \backslash\left\{p_{0}\right\}$, that is, if $p_{0} \notin \widehat{A}$, then $A=\widehat{A}$, otherwise $A$ is obtained by removing $p_{0}$ from $\widehat{A}$.

\section{Csirmaz Framework for Proving Lower Bounds and Its Limitations}

\subsection{Csirmaz Framework for Proving Lower Bounds}

Csirmaz [18] has proved the best known lower bounds on the size of the shares in secret-sharing schemes. Towards this goal, he presented a framework for proving lower bounds and showed how to implement this framework to prove lower bounds for a specific access structure. The idea of the framework of Csirmaz is to construct a linear program such that lower bounds on the value of the objective function in this program imply lower bounds on the share size. Specifically, given an access structure $\mathcal{A}$ and a secret-sharing scheme realizing it, define the function $f(\widehat{A})=H\left(S_{\widehat{A}}\right) / H(S)$ for every $\widehat{A} \subseteq P \cup\left\{p_{0}\right\}$. The correctness and privacy of the secret-sharing scheme can be translated to constrains on the function $f$. Namely, (1) if $A \in \mathcal{A}$, then $f\left(A \cup\left\{p_{0}\right\}\right)=f(A)$, and (2) if $A \notin \mathcal{A}$, then $f\left(A \cup\left\{p_{0}\right\}\right)=f(A)+1$. Proving lower bounds on the size of the shares is equivalent to proving that any $n$ random variables $S_{1}, \ldots, S_{n}$ (i.e., shares) satisfying the above equalities imply that $\sum_{i=1}^{n} H\left(S_{i}\right)$ is large. 
These constrains are translated to a linear program using known properties of the entropy function, namely, information inequalities. That is, we get a set of linear inequalities, where we want to minimize $\sum_{i=1}^{n} f\left(\left\{p_{i}\right\}\right)$.

Csirmaz has constructed an access structure $\mathcal{A}$ that implies a linear program in which $\sum f\left(\left\{p_{i}\right\}\right)=\Omega\left(n^{2} / \log n\right)$, thus, for at least one party $f\left(\left\{p_{i}\right\}\right)=$ $\Omega(n / \log n)$. This implies that in every secret-sharing scheme realizing $\mathcal{A}$ with an $\ell$-bit secret, the share of at least one party is an $\Omega(\ell \cdot n / \log n)$-bit string. We next formally define and describe Csirmaz's framework.

Definition 4. Given a secret sharing scheme over $n$ parties, define the function $f: 2^{P \cup\left\{p_{0}\right\}} \rightarrow \mathbb{R}$ as follow: $f(\widehat{A})=H\left(S_{\widehat{A}}\right) / H(S)$ for every $\widehat{A} \subseteq P \cup\left\{p_{0}\right\}$.

The properties of the entropy function implies that $f$ is a polymatroid as defined below.

Definition 5. Let $Q$ be a finite set, and $g: 2^{Q} \rightarrow \mathbb{R}$ be a function assigning real numbers to subsets of $Q$. The system $(Q, g)$ is a polymatroid if $g$ satisfies the following conditions:

non-negative: $g(A) \geq 0$ for all $A \subseteq Q$ and $g(\emptyset)=0$,

monotone: if $A \subseteq B \subseteq Q$, then $g(A) \leq g(B)$,

submodular: $g(A)+g(B) \geq g(A \cup B)+g(A \cap B)$ for every $A, B \subseteq Q$.

Proposition 1 ([25]). The function $f$ defined in Definition 4 is a polymatroid.

Combining Proposition 1 and the properties of secret-sharing scheme we get:

Proposition 2. The function $f$ defined in Definition 4 satisfies the following additional inequalities for every sets $A, B \subseteq P$ :

1. If $A \subseteq B, A \notin \mathcal{A}$, and $B \in \mathcal{A}$, then $f(B) \geq f(A)+1$,

2. If $A \in \mathcal{A}, B \in \mathcal{A}$, but $A \cap B \notin \mathcal{A}$, then $f(A)+f(B) \geq f(A \cap B)+f(A \cup B)+1$.

\subsection{Limitation of Shannon Inequalities}

Csirmaz 18 has proved that using his framework with only Shannon inequalities (which were the only information inequalities known when he published his result) one cannot prove lower bounds better than $\Omega(n)$. That is, his lower bound is the best possible up to a factor of $\log n$ using only Shannon inequalities.

In this section we explain how Csirmaz proved this limitation. Since Csirmaz proved his result in 1994, some non-Shannon information inequalities were discovered. In Section [6] we will show that these inequalities cannot prove better lower bounds than $\Omega(n)$ using Csirmaz's framework.

Theorem 1. Given any access structure $\mathcal{A}$ on the $n$-element set $P$, there is a polymatroid $\widehat{g}: 2^{P \cup\left\{p_{0}\right\}} \rightarrow \mathbb{R}$ so that

1. For every $A \subseteq P, \widehat{g}\left(A \cup\left\{p_{0}\right\}\right)=\widehat{g}(A)$ if $A \in \mathcal{A}$ and $\widehat{g}\left(A \cup\left\{p_{0}\right\}\right)=\widehat{g}(A)+1$ if $A \notin \mathcal{A}$.

2. $\widehat{g}$ satisfies the conditions of Proposition $\mathbf{2}$.

3. $\widehat{g}\left(\left\{p_{i}\right\}\right) \leq n$ for every $p_{i} \in P$. 
In order to prove this theorem, Csirmaz has defined a polymatroid $\widehat{g}$ that, on one hand, satisfies all the conditions and, on the other hand, $\widehat{g}\left(\left\{p_{i}\right\}\right)=n$. In other words, Csirmaz has shown that for every access structure the linear program has a small solution.

Definition 6 (The Csirmaz function). Let $n \in \mathbb{N}$. Define the the Csirmaz function $C_{n}:\{0, \ldots, n\} \rightarrow \mathbb{N}$ as follows

$$
\mathcal{C}_{n}(k) \stackrel{\text { def }}{=} n+(n-1)+\ldots+(n-k+1)=n k+\frac{k}{2}-\frac{k^{2}}{2} .
$$

To prove Theorem 11. Csirmaz defined $g: 2^{P} \rightarrow \mathbb{N}$ as $g(A) \stackrel{\text { def }}{=} \mathcal{C}_{n}(|A|)$. Next, he extended $g$ to $\widehat{g}: 2^{P \cup\left\{p_{0}\right\}} \rightarrow \mathbb{N}$, where for every $A \subseteq P$ he defined $\widehat{g}(A)=g(A)$, and $\widehat{g}\left(A \cup\left\{p_{0}\right\}\right)=g(A)$ if $A \in \mathcal{A}$, and $\widehat{g}\left(A \cup\left\{p_{0}\right\}\right)=g(A)+1$ if $A \notin \mathcal{A}$. It can be checked that $\widehat{g}$ satisfies the conditions of the theorem. The Csirmaz function is universal; it is used to construct a polymatroid for every access structure. We next prove that any such universal function is at least as large as the Csirmaz function. This lemma sheds some light why Csirmaz chose this function.

Lemma 1. Let $y_{n}:\{0, \ldots, n\} \rightarrow \mathbb{R}$ be a function satisfying the following inequalities:

1. If $A \subseteq B \subseteq Q$, then $y_{n}(|B|) \geq y_{n}(|A|)+1$ and $y_{n}(0)=0$,

2. If $A$ and $B$ are subsets of $Q$ such that $A \nsubseteq B$ and $B \nsubseteq A$, then $y_{n}(|A|)+$ $y_{n}(|B|) \geq y_{n}(|A \cap B|)+y_{n}(|A \cup B|)+1$.

The Csirmaz Function $\mathcal{C}_{n}(k)$ is the minimal function that satisfies these requirements, i.e., for each $1 \leq k \leq n, \mathcal{C}_{n}(k) \leq y_{n}(k)$.

Proof. Let $A, B$ be two sets of $k$ elements each that are different in exactly one element. Thus, $|A \cap B|=k-1$ and $|A \cup B|=k+1$. From Item (2) in the lemma, for each $0 \leq k \leq n$

$$
y_{n}(k)-y_{n}(k-1) \geq y_{n}(k+1)-y_{n}(k)+1 .
$$

This implies that $y_{n}(k)-y_{n}(k-1) \geq y_{n}(n)-y_{n}(n-1)+n-k$ for every $0 \leq k \leq n$. By Item (11) in the lemma, $y_{n}(|B|) \geq y_{n}(|A|)+1$. Thus, $y_{n}(n)-y_{n}(n-1) \geq 1$. Therefore,

$$
y_{n}(k)-y_{n}(k-1) \geq n-k+1
$$

By the requirement in the lemma $y_{n}(0)=0$, thus, Inequality (II) with $k=1$ implies $y_{n}(1) \geq n=\mathcal{C}_{n}(1)$. By induction and by (II), $y_{n}(k) \geq y_{n}(k-1)+n-$ $k+1 \geq \mathcal{C}_{n}(k-1)+n-k+1=\mathcal{C}_{n}(k)$.

\section{When Can Information Inequalities Help?}

In this section, we will define when information inequalities can help in improving lower bounds beyond $\Omega(n)$. We start with some notation; using this notation 
we will define two quantities for an information inequality, $\Delta$ and $\Lambda$. These quantities are used to define when an information inequality can help.

Notation 2. Let $A_{1}, \ldots, A_{m}$ be $m$ (not necessarily disjoint) sets. For $I \subseteq[m]$, denote $A_{I}=\bigcup_{i \in I} A_{i}$.

Let $\sum_{I \subseteq[m]} \alpha_{I} H\left(X_{I}\right) \geq 0$ be an information inequality. Given an access structure $\mathcal{A}$, we fix some secret-sharing scheme realizing it. Therefore, the function $f(\widehat{A})=H\left(S_{\widehat{A}}\right) / H(S)$ where $\widehat{A} \subseteq P \cup\left\{p_{0}\right\}$ is well defined. Then, for every sets $\widehat{A}_{1}, \ldots, \widehat{A}_{m} \subseteq P \cup\left\{p_{0}\right\}$, the following inequality is valid $\sum_{I \subseteq[m]} \alpha_{I} f\left(\widehat{A}_{I}\right) \geq 0$. Recall that for every $1 \leq i \leq m$, we defined $A_{i}=\widehat{A}_{i} \backslash\left\{p_{0}\right\}$. Using this notation, $f\left(\widehat{A}_{I}\right)=f\left(A_{I}\right)+1$ if $p_{0} \in \widehat{A}_{I}$ and $A_{I} \notin \mathcal{A}$, otherwise, $f\left(\widehat{A}_{I}\right)=f\left(A_{I}\right)$.

Definition 7. For an information inequality $\sum_{I \subseteq[m]} \alpha_{I} H\left(X_{I}\right) \geq 0$, an access structure $\mathcal{A}$, and sets $\widehat{A}_{1}, \ldots, \widehat{A}_{m}$, define $\Delta$ as $\Delta \stackrel{\text { def }}{=}-\sum_{I: p_{0} \in \widehat{A}_{I} ; A_{I} \notin \mathcal{A}} \alpha_{I}$.

Claim 1. Let $\widehat{A}_{1}, \ldots, \widehat{A}_{m}$ be $m$ sets, $\sum_{I \subseteq[m]} \alpha_{I} H\left(X_{I}\right) \geq 0$ be an information inequality, and $\mathcal{A}$ be an access structure. Then, $\sum_{I \subseteq[m]} \alpha_{I} f\left(A_{I}\right) \geq \Delta$.

Proof. Applying the rules $f\left(\widehat{A}_{I}\right)=f\left(A_{I}\right)$ if $p_{0} \notin \widehat{A}_{I}$ or $A_{I} \in \mathcal{A}$, and $f\left(\widehat{A}_{I}\right)=$ $f\left(A_{I}\right)+1$ otherwise, the inequality $\sum_{I \subseteq[m]} \alpha_{I} f\left(\widehat{A}_{I}\right) \geq 0$ implies

$$
\begin{aligned}
\sum_{I \subseteq[m]} \alpha_{I} f\left(\widehat{A}_{I}\right) & =\sum_{I: p_{0} \notin \widehat{A}_{I} \vee A_{I} \in \mathcal{A}} \alpha_{I} f\left(A_{I}\right)+\sum_{I: p_{0} \in \widehat{A}_{I} \wedge A_{I} \notin \mathcal{A}} \alpha_{I}\left(f\left(A_{I}\right)+1\right) \\
& =\sum_{I \subseteq[m]} \alpha_{I} f\left(A_{I}\right)-\Delta \geq 0 .
\end{aligned}
$$

Observe that $\Delta$ can be negative, positive, or equal to zero, but, as we will see later, the information inequality can be useful only when $\Delta>0$.

Definition 8. Let $\sum_{I \subseteq[m]} \alpha_{I} H\left(X_{I}\right) \geq 0$ be an information inequality. For sets $A_{1}, \ldots, A_{m} \subseteq P$ define $\Lambda$ as $\Lambda \stackrel{\text { def }}{=} \sum_{I \subseteq[m]} \alpha_{I} \mathcal{C}_{n}\left(\left|A_{I}\right|\right)$.

For every $I \subseteq[m]$, the size $\left|A_{I}\right|$ depends on some of the sizes of the intersections between the sets $A_{1}, \ldots, A_{m}$. Therefore, we define additional notation in order to represent these intersections. For an illustration of this notation see Fig. [1

Notation 3. Let $A_{1}, \ldots, A_{m}$ be $m$ (not necessarily disjoint) sets. Denote $\delta_{I} \stackrel{\text { def }}{=}$ $\bigcap_{i \in I} A_{i} \backslash \bigcup_{i \notin I} A_{\{i\}}$ and $t_{I} \stackrel{\text { def }}{=}\left|\delta_{I}\right|$ for $I \subseteq[m]$. In addition, for $\mathcal{I} \subseteq 2^{[m]}$, denote $\delta_{\mathcal{I}} \stackrel{\text { def }}{=} \bigcup_{I \in \mathcal{I}} \delta_{I}$.

Observation 1. $\delta_{J} \subseteq A_{i}$ if and only if $i \in J$, that is, $A_{i}=\cup_{i \in J} \delta_{J}$ and $A_{I}=$ $\cup_{i \in I} A_{i}=\cup_{I \cap J \neq \emptyset} \delta_{J}$. 


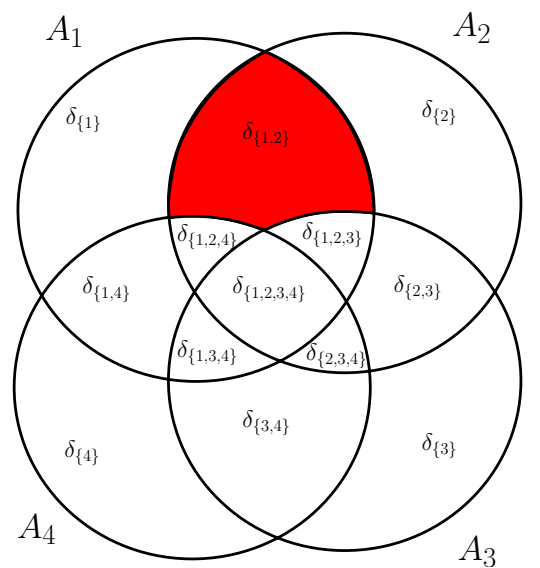

(a) $\delta_{\{1,2\}}=\left(A_{1} \cap A_{2}\right) \backslash\left(A_{3} \cup A_{4}\right)$.

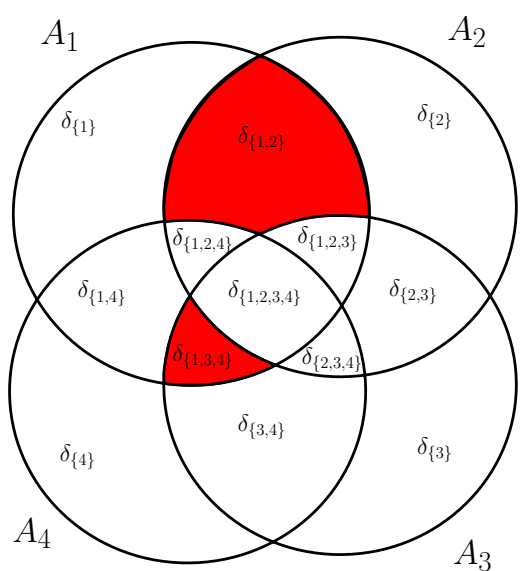

(b) $\delta_{\{\{1,2\},\{1,3,4\}\}}=\delta_{\{1,2\}} \cup \delta_{\{1,3,4\}}$.

Fig. 1. An illustration of Notation [3] for $m=4$. For clarity of the illustration, we assume that $\delta_{\{2,4\}}=\delta_{\{1,3\}}=\emptyset$.

Csirmaz has suggested a specific function defined in Definition 6 in order to show the limitations of Shannon information inequalities. We will prove in Lemma 4 that any information inequality remains valid after plugging in the Csirmaz function. That is, if $\sum_{I \subseteq[m]} \alpha_{I} H\left(X_{I}\right) \geq 0$ is an information inequality, then $\sum_{I \subseteq[m]} \alpha_{I} \mathcal{C}_{n}\left(\left|A_{I}\right|\right) \geq 0$. So, our only hope is that $\Delta$ is "big" for some sets $\widehat{A}_{1}, \ldots, \widehat{A}_{m} \subseteq P \cup\left\{p_{0}\right\}$ and the corresponding sets $A_{1}, \ldots, A_{m} \subseteq P$, but, $\Lambda=$ $\sum_{I \subseteq[m]} \alpha_{I} \mathcal{C}_{n}\left(\left|A_{I}\right|\right)$ is negative (or "small"). If this condition does not hold, then the inequality cannot help.

Definition 9. We say that an information inequality $\sum_{I \subseteq[m]} \alpha_{I} H\left(X_{I}\right) \geq 0$ can at most $\gamma$-help if $\Delta \leq \gamma \Lambda$ for every sets $\widehat{A}_{1}, \ldots, \widehat{A}_{m} \subseteq P \cup\left\{p_{0}\right\}$ and for every access structure $\mathcal{A}$, where $\Delta=-\sum_{I: p_{0} \in \widehat{A}_{I} ; A_{I} \notin \mathcal{A}} \alpha_{I}$ and $\Lambda=\sum_{I \subseteq[m]} \alpha_{I} \mathcal{C}_{n}\left(\left|A_{I}\right|\right)$.

Theorem 2. Let $\gamma>0$ be a constant. Consider a collection of information inequalities, where each information inequality in the collection can at most $\gamma$ help. Then, this collection of information inequalities cannot help improving the lower bounds beyond $\gamma n$ even if all inequalities are used simultaneously.

Proof. Consider an access structure $\mathcal{A}$ and the "huge" linear program obtained for this access structure by applying each information inequality to every choice of subsets of the parties. We take the polymatroid $g\left(A_{I}\right)=\gamma \mathcal{C}_{n}\left(\left|A_{I}\right|\right)$, and we get a solution that satisfies each inequality in the program, where $g\left(\left\{p_{i}\right\}\right)=\gamma n$.

When dealing with a finite collection of information inequalities, one can use a rougher notion than an information inequality that can at most $\gamma$-help. 
Definition 10. We say that an information inequality $\sum_{I \subseteq[m]} \alpha_{I} H\left(X_{I}\right) \geq 0$ cannot help (in improving the lower bounds beyond $\Omega(n)$ ) if for every sets $\widehat{A}_{1}, \ldots, \widehat{A}_{m} \subseteq P \cup\left\{p_{0}\right\}$ and for every access structure $\mathcal{A}$, if $\Delta>0$ then $\Lambda>0$.

Observation 2. Let $\sum_{I \subseteq[m]} \alpha_{I} H\left(X_{I}\right) \geq 0$ be an information inequality that cannot help. Observe that $\Delta=-\sum_{I: p_{0} \in \widehat{A}_{I} ; A_{I} \notin \mathcal{A}} \alpha_{I} \geq-\sum_{I: p_{0} \in \widehat{A}_{I} ; A_{I} \notin \mathcal{A} ; \alpha_{I}<0} \alpha_{I}$. In addition, using Lemma 3 (proved later), if $\Lambda>0$ then there exists a constant $\beta>0$ that depends only on the coefficients of the information inequality (and, therefore, independent of the access structure and the number of parties in the access structure) such that $\Lambda \geq \beta$ Q Thus, the information inequality can at most $\gamma$-help for some constant $\gamma>0$. If we consider a finite collection of information inequalities, such that each inequality in the collection cannot help, then there is a constant $\gamma>0$ such that each inequality in the collection can at most $\gamma$-help, and we can apply Theorem [2. Therefore, when dealing with a finite collection of information inequalities, we will check that each inequality in the collection cannot help; this is easier than calculating the maximal $\gamma$ for each inequality.

\section{Examples of Information Inequalities That Cannot Help}

In this section, we demonstrate our method for proving that an information inequality cannot help by considering two example. First, we will demonstrate the calculations and the technique that we will use later on a simple Shannon inequality with two random variables. The fact that this inequality cannot help follows from Csirmaz's proof that using only Shannon inequalities one cannot prove better lower bounds. We reprove this result in order to supply a simple example of our method.

We consider the inequality $f\left(\widehat{A}_{1}\right)+f\left(\widehat{A}_{2}\right) \geq f\left(\widehat{A}_{1} \cup \widehat{A}_{2}\right)+f\left(\widehat{A}_{1} \cap \widehat{A}_{2}\right)$ for two sets $\widehat{A}_{1}, \widehat{A}_{2} \subseteq P \cup\left\{p_{0}\right\}$. This inequality follows from the fact that the conditional mutual information is non-negative. We should calculate $\Lambda=\mathcal{C}_{n}\left(\left|A_{1}\right|\right)+$ $\mathcal{C}_{n}\left(\left|A_{2}\right|\right)-\mathcal{C}_{n}\left(\left|A_{1} \cup A_{2}\right|\right)-\mathcal{C}_{n}\left(\left|A_{1} \cap A_{2}\right|\right)$. By Obseration [1 $\left|A_{1}\right|=t_{1}+t_{1,2}$, $\left|A_{2}\right|=t_{2}+t_{1,2},\left|A_{1} \cup A_{2}\right|=t_{1}+t_{1,2}+t_{2}$, and $\left|A_{1} \cap A_{2}\right|=t_{1,2} 3$ Furthermore, $n=t_{1}+t_{1,2}+t_{2}$. Therefore, for every $A_{1}, A_{2} \subseteq P$

$$
\begin{aligned}
\mathcal{C}_{n}\left(\left|A_{1}\right|\right) & +\mathcal{C}_{n}\left(\left|A_{2}\right|\right)-\mathcal{C}_{n}\left(\left|A_{1} \cup A_{2}\right|\right)-\mathcal{C}_{n}\left(\left|A_{1} \cap A_{2}\right|\right) \\
= & \left(t_{1}+t_{1,2}\right)\left[\left(t_{1}+t_{1,2}+t_{2}\right)+\frac{1}{2}-\frac{\left(t_{1}+t_{1,2}\right)}{2}\right] \\
& +\left(t_{2}+t_{1,2}\right)\left[\left(t_{1}+t_{1,2}+t_{2}\right)+\frac{1}{2}-\frac{\left(t_{2}+t_{1,2}\right)}{2}\right] \\
& -\left(t_{1}+t_{1,2}+t_{2}\right)\left[\left(t_{1}+t_{1,2}+t_{2}\right)+\frac{1}{2}-\frac{\left(t_{1}+t_{1,2}+t_{2}\right)}{2}\right] \\
& -t_{1,2}\left[\left(t_{1}+t_{1,2}+t_{2}\right)+\frac{1}{2}-\frac{\left(t_{1,2}\right)}{2}\right]=t_{1} t_{2} .
\end{aligned}
$$

\footnotetext{
${ }^{2}$ The value of $\beta$ can be calculated by assigning $t_{I}=1$ whenever $t_{I}>0$.

${ }^{3}$ For simplicity of our notation, in the rest of the paper we sometimes write $t_{1,2}$ instead of $t_{\{1,2\}}$ (and similarly for other sets).
} 
Assume that $p_{0} \in \widehat{A}_{1}, \widehat{A}_{2}$. Thus, $p_{0} \in \widehat{A}_{1} \cup \widehat{A}_{2}, \widehat{A}_{1} \cap \widehat{A}_{2}$. Before calculating $\Delta$ we have to decide which sets are in the access structure. If $A_{1} \cup A_{2} \notin \mathcal{A}$, then also $A_{1}, A_{2}, A_{1} \cap A_{2} \notin \mathcal{A}$. Thus, $f\left(A_{1} \cup\left\{p_{0}\right\}\right)=f\left(A_{1}\right)+1, f\left(A_{2} \cup\left\{p_{0}\right\}\right)=f\left(A_{2}\right)+1$, $f\left(A_{1} \cup A_{2} \cup\left\{p_{0}\right\}\right)=f\left(A_{1} \cup A_{2}\right)+1$, and $f\left(A_{1} \cap A_{2} \cup\left\{p_{0}\right\}\right)=f\left(A_{1} \cap A_{2}\right)+1$. Therefore, $\Delta=0$ and the inequality cannot help using these selections. However, if $A_{1}, A_{2} \in \mathcal{A}$, but $A_{1} \cap A_{2} \notin \mathcal{A}$, then $f\left(A_{1} \cup\left\{p_{0}\right\}\right)=f\left(A_{1}\right), f\left(A_{2} \cup\left\{p_{0}\right\}\right)=f\left(A_{2}\right)$, $f\left(A_{1} \cup A_{2} \cup\left\{p_{0}\right\}\right)=f\left(A_{1} \cup A_{2}\right)$, and $f\left(\left(A_{1} \cap A_{2}\right) \cup\left\{p_{0}\right\}\right)=f\left(A_{1} \cap A_{2}\right)+1$. Therefore, $\Delta=1>0$ as needed. But the selection of $A_{1}, A_{2} \in \mathcal{A}$ and $A_{1} \cap A_{2} \notin \mathcal{A}$ implies $A_{1} \backslash\left(A_{1} \cap A_{2}\right), A_{2} \backslash\left(A_{1} \cap A_{2}\right) \neq \emptyset$ which means that $t_{1}>0$ and $t_{2}>0$, thus, $\Lambda=t_{1} \cdot t_{2} \geq 1>0$ as well. In other words using these selections the inequality cannot help. Moreover, every other set of selections cannot help to achieve $\Delta>0$ while $\Lambda=0$.

To conclude, given an information inequality we want $\Delta>0$ while $\Lambda=0$. By different choices of which sets are in the access structure and which sets contain the dealer we get different values of $\Delta$. We want choices that maximize $\Delta$. However, notice that by choosing, for example, $A_{1} \in \mathcal{A}$ while $A_{2} \notin \mathcal{A}$, we must have that $A_{1} \backslash A_{2} \neq \emptyset$. Thus, the choices of which sets are in the access structure force that certain sets are non-empty, which might imply that $\Lambda>0$.

\subsection{The Zhang and Yeung Information Inequality Cannot Help}

We next consider the Zhang and Yeung information inequality [47] - the first Non-Shannon inequality that was discovered - and prove that this inequality cannot help in proving lower bounds of $\omega(n)$.

Theorem 3 (The Zhang and Yeung Information Inequality [47, Theorem 3]). For every four discrete random variables $X_{1}, X_{2}, X_{3}$, and $X_{4}$ the following inequality holds:

$$
\begin{gathered}
3\left[H\left(X_{3} X_{4}\right)+H\left(X_{2} X_{4}\right)+H\left(X_{2} X_{3}\right)\right]+H\left(X_{1} X_{3}\right)+H\left(X_{1} X_{2}\right)-H\left(X_{4}\right) \\
-2\left[H\left(X_{3}\right)+H\left(X_{2}\right)\right]-H\left(X_{1} X_{4}\right)-4 H\left(X_{2} X_{3} X_{4}\right)-H\left(X_{1} X_{2} X_{3}\right) \geq 0 .
\end{gathered}
$$

For every secret-sharing scheme and for every four sets $\widehat{A}_{1}, \widehat{A}_{2}, \widehat{A}_{3}, \widehat{A}_{4} \subseteq P \cup\left\{p_{0}\right\}$ we can consider the random variables $X_{i}=S_{\widehat{A}_{i}}$ for $i=1, \ldots, 4$. Thus,

$$
\begin{gathered}
3\left[f\left(\widehat{A}_{3} \widehat{A}_{4}\right)+f\left(\widehat{A}_{2} \widehat{A}_{4}\right)+f\left(\widehat{A}_{2} \widehat{A}_{3}\right)\right]+f\left(\widehat{A}_{1} \widehat{A}_{3}\right)+f\left(\widehat{A}_{1} \widehat{A}_{2}\right)-f\left(\widehat{A}_{4}\right) \\
-2\left[f\left(\widehat{A}_{3}\right)-f\left(\widehat{A}_{2}\right)\right]-f\left(\widehat{A}_{1} \widehat{A}_{4}\right)-4 f\left(\widehat{A}_{2} \widehat{A}_{3} \widehat{A}_{4}\right)-f\left(\widehat{A}_{1} \widehat{A}_{2} \widehat{A}_{3}\right) \geq 0 .
\end{gathered}
$$

By choosing which sets contain the dealer and which sets are in the access structure we get different values of $\Delta$. We next apply the Csirmaz function on Inequality (B). We use the same process described above on each one of the terms of (3). After simplifications, we get the following polynomial $\Lambda$, which is a multivariate polynomial whose variables are $\left\{t_{I}: I \subseteq[m]\right\}$. 


$$
\begin{aligned}
& \overbrace{t_{1,2,3}+t_{1,2,3}^{2}}^{2}+t_{1} t_{1,2,3}+t_{1,2} t_{1,2,3}+\overbrace{t_{1,2,4}+t_{1,2,4}^{2}}+2 t_{1} t_{1,2,4}+2 t_{1,2} t_{1,2,4}+t_{1,2} t_{1,3} \\
& +\overbrace{1,2,3} t_{1,3}+\overbrace{t_{1,3,4}+t_{1,3,4}^{2}}^{t_{1,4}+t_{1,4}^{2}} \\
& +2 t_{1,2,4} t_{1,4}+2 t_{1,3} t_{1,4}+t_{1,3,4} t_{1,3,4}+2 t_{1,3} t_{1,3,4}+t_{1,2,3} t_{2}+2 t_{1,2,4} t_{1,4}+2 t_{1,2} t_{1,4}+\overbrace{t_{1,3} t_{2}}+2 t_{1,4} t_{2}+t_{1,2} t_{2,3} \\
& +\overbrace{t_{2,3}+t_{2,3}^{2}}^{t_{2,4}+t_{2,4}^{2}} \frac{t_{1} t_{2,3}}{2}+t_{1,2,3} t_{2,3}+t_{1,3} t_{2,3}+2 t_{2} t_{2,3}+t_{2,4}+t_{2} t_{2,4} \\
& +2 t_{1,2} t_{2,4}+2 t_{1,2,4} t_{2,4}+2 t_{1,4} t_{2,4}+t_{1,2} t_{3}+t_{1,2,3} t_{3}+2 t_{1,3,4} t_{3}+2 t_{1,4} t_{3}+2 t_{2} t_{3} \\
& +2 t_{2,3} t_{3}+\frac{\overbrace{3,4}+t_{3,4}^{2}}{2}+2 t_{1} t_{3,4}+2 t_{1,3} t_{3,4}+2 t_{1,3,4} t_{3,4}+2 t_{1,4} t_{3,4}+t_{3} t_{3,4}++t_{1} t_{4} \\
& +2 t_{1,2} t_{4}+2 t_{1,2,4} t_{4}+2 t_{1,3} t_{4}+2 t_{1,3,4} t_{4}+t_{1,4} t_{4}+t_{2} t_{4}+t_{2,4} t_{4}+t_{3} t_{4}+t_{3,4} t_{4} .
\end{aligned}
$$

After applying the Csirmaz function we get a polynomial of degree 2 such that all its coefficients are non-negative. We are looking for the following situation: $\Lambda=0$ while $\Delta>0$. Since all coefficients are non-negative and $t_{I} \geq 0$ for every $I \subseteq[\mathrm{m}]$, the value of $\Lambda$ is zero if every monomial in $\Lambda$ is zero. In particular, every term $\beta \cdot t_{I}$ or $\beta \cdot t_{I}^{2}$ in $\Lambda$ has to be equal to zero. If the coefficient $\beta$ is positive, then $t_{I}=0$ must hold. Thus, $t_{1,2,3}=t_{1,2,4}=t_{1,3,4}=t_{1,4}=t_{2,3}=t_{2,4}=t_{3,4}=0$. Let $\Lambda^{\prime}$ be the polynomial after setting these variables to be zero, that is,

$$
\Lambda^{\prime}=t_{1,2} t_{1,3}+t_{1,3} t_{2}+t_{1,2} t_{3}+2 t_{2} t_{3}+t_{1} t_{4}+2 t_{1,2} t_{4}+2 t_{1,3} t_{4}+t_{2} t_{4}+t_{3} t_{4} .
$$

The polynomial $\Lambda^{\prime}$ should be zero, therefore, in the inequality above one of the variables (i.e., set size) in each monomial has to be zero (e.g., $t_{1,2}=0$ or $t_{1,3}=0$ ).

We use a brute-force algorithm for checking if it is possible that $\Delta>0$ while $\Lambda=0$. We have two decisions to make:

- For each $i \in\{1, \ldots, 4\}$ we should decide if $p_{0} \in \widehat{A}_{i}$ or not.

- We have to decide which sets are in the access structure. Specifically, for each $I \subseteq[m]$ such that $\alpha_{I} \neq 0$ in the information inequality, we need to decide whether $A_{I} \notin \mathcal{A}$ or $A_{I} \in \mathcal{A}$. These decisions should be consistent with the constrains that some sets $\delta_{J}$ are of size zero.

Example 1. Assume that $A_{4}$ is the only minimal set in the in the access structure. Thus, the sets that are in the access structure are exactly those that include $A_{4}$. We add the dealer to $\widehat{A}_{2}$ and do not add it to any other set. After committing to these decisions we compute $\Delta$ as specified in Definition $\mathbf{7} \Delta=-\sum_{2 \in I, 4 \notin I} \alpha_{I}=$ $-(3+1-2-1)=-1<0$. Thus, these decisions cannot help.

Example 2. Assume that $A_{\{1,2\}}$ and $A_{\{2,3\}}$ are the only minimal sets in the in the access structure. This means that the sets that are in the access structure are exactly those that include $A_{\{1,2\}}$ or $A_{\{2,3\}}$. For example, $A_{\{1,2,3\}} \in \mathcal{A}$. We also add the dealer to every $\widehat{A}_{i}, 1 \leq i \leq 4$. After committing to these two decisions we compute $\Delta=-\sum_{\{1,2\} \nsubseteq I \wedge\{1,3\} \nsubseteq I} \alpha_{I}=-(3+3+3-1-2-2-1-4)=1>0$. Observe that $\Delta>0$ as needed. But, $A_{\{1,2\}} \in \mathcal{A}$ while $A_{\{1,3\}} \notin \mathcal{A}$. This means 
that $A_{\{1,2\}} \backslash A_{\{1,3\}}=\delta_{\{\{2\},\{2,4\}\}} \neq \emptyset$. However, we have set $t_{2,4}=0$, thus, $t_{2} \neq 0$. In a similar way, $A_{\{2,3,4\}} \in \mathcal{A}$ while $A_{\{2,3\}} \notin \mathcal{A}$. This means that $A_{\{2,3,4\}} \backslash A_{\{2,3\}}=\delta_{\{\{4\},\{1,4\}\}} \neq \emptyset$. However, we have set $t_{1,4}=0$, thus, $t_{4} \neq 0$. Combining these two constraints we get $t_{2} \cdot t_{4}>0$, which implies $\Lambda>0$. Thus, as before, these decisions cannot help.

We have written a computer program that checks all the possibilities for including the dealer in the sets and for which sets are in the access structure. The computer program showed that for each possible combination either $\Delta \leq 0$ or $\Lambda>0$ (or both). This means that the Csirmaz function is still a solution to the linear program and this inequality cannot help.

\section{All Known Information Inequalities Cannot Help}

In this section we describe an algorithm that checks if an information inequality cannot help. We executed this algorithm on all known information inequalities, except for two infinite collections of inequalities, and verified that they cannot help. Thereafter, we consider the two known infinite collections of information inequalities and show that they can at most $\gamma$-help for some constant $\gamma>0$. Before presenting these results, we show how to compute the polynomial $\Lambda$ efficiently and analyze its properties.

\subsection{Properties of the Polynomial $\Lambda$}

For every information inequality $\sum_{I \subseteq[m]} \alpha_{I} H\left(X_{I}\right) \geq 0$ and for every sets $A_{1}, \ldots$, $A_{m}$ we consider the quantity $\Lambda=\sum_{I \subseteq[m]} \alpha_{I} \mathcal{C}_{n}\left(\left|A_{I}\right|\right)$. By Obseration [1] $\left|A_{I}\right|=$ $\sum_{I \cap J \neq \emptyset} t_{J}$. Thus, we consider $\Lambda=\sum_{I \subseteq[m]} \alpha_{I} \mathcal{C}_{n}\left(\sum_{I \cap J \neq \emptyset} t_{J}\right)$ as a polynomial in the variables $\left\{t_{J}\right\}_{J \subseteq[m]}$. We start with proving a property of information inequalities that we use in the analysis of our algorithm.

Lemma 2. Let $\sum_{I} \alpha_{I} H\left(X_{I}\right) \geq 0$ be an information inequality. Then, for every $J \subseteq[m], \sum_{I \cap J \neq \emptyset} \alpha_{I} \geq 0$.

Proof. Define a random variable $Y$ which is uniformly distributed in $\{0,1\}$; in particular $H(Y)=1$. Now define $X_{1}, \ldots, X_{m}$, where $X_{j}=Y$ iff $j \in J$ and $X_{j}=0$ otherwise (that is, in the latter case $X_{j}$ is a deterministic variable whose entropy is 0$)$. This implies that $H\left(X_{I}\right)=1$ iff $I \cap J \neq \emptyset$ and $H\left(X_{I}\right)=0$ otherwise. Since the information inequality holds for every random variables, the lemma follows.

Lemma 3. For every information inequality the polynomial $\Lambda$ is a multivariate polynomial with total degree 2. Furthermore, the coefficient of every monomial in $\Lambda$ is non-negative and can be efficiently calculated from the information inequality (without applying the Csirmaz function).

Proof. The fact that the polynomial $\Lambda$ is a multivariate polynomial with total degree 2 can be deduced from the structure of the Csirmaz function (see Definition [6), that is, $\Lambda$ is a sum of polynomials $\mathcal{C}_{n}\left(\left|A_{I}\right|\right)=\mathcal{C}_{n}\left(\sum_{I \cap J \neq \emptyset} t_{J}\right)$, where 
$\mathcal{C}_{n}(k)$ is polynomial of degree 2 . Next, we compute the coefficients of $\Lambda$. Recall that $n=\sum_{I \subseteq[m]} t_{I}$.

$$
\begin{aligned}
\Lambda= & \sum_{I \subseteq[m]} \alpha_{I} \mathcal{C}_{n}\left(\left|A_{I}\right|\right)=\sum_{I \subseteq[m]} \alpha_{I}\left[n\left|A_{I}\right|+\frac{\left|A_{I}\right|}{2}-\frac{\left|A_{I}\right|^{2}}{2}\right] \\
= & \sum_{I \subseteq[m]} \alpha_{I}\left(\sum_{J: I \cap J \neq \emptyset} t_{J}+\sum_{J: I \cap J=\emptyset} t_{J}\right)\left(\sum_{J: I \cap J \neq \emptyset} t_{J}\right)+\sum_{I \subseteq[m]} \alpha_{I} \frac{\sum_{J: I \cap J \neq \emptyset} t_{J}}{2} \\
& -\sum_{I \subseteq[m]} \alpha_{I} \frac{\left(\sum_{J: I \cap J \neq \emptyset} t_{J}\right)^{2}}{2} \\
= & \sum_{I \subseteq[m]} \alpha_{I}\left(\frac{\sum_{J: I \cap J \neq \emptyset} t_{J}+\left(\sum_{J: I \cap J \neq \emptyset} t_{J}\right)^{2}}{2}+\sum_{J: I \cap J \neq \emptyset} t_{J} \cdot \sum_{J: I \cap J=\emptyset} t_{J}\right) .
\end{aligned}
$$

We can now compute the coefficients of the monomials of the polynomial $\Lambda$ :

1. $\beta t_{J}$ : In this case $\beta=\frac{\sum_{I \cap J \neq \emptyset} \alpha_{I}}{2}$, i.e., the sum of the coefficients of sets that include $\delta_{J}$. By Lemma 2 this sum is non negative.

2. $\beta t_{J}^{2}$ : In this case $\beta=\frac{\sum_{I \cap J \neq \emptyset} \alpha_{I}}{2}$, again, this is the sum of the coefficients of sets that include $\delta_{J}$.

3. $\beta t_{J} t_{K}$ : In this case $\beta=\sum_{I: I \cap(J \cup K) \neq \emptyset} \alpha_{I}$. That is, $\beta$ is the sum of coefficients of sets that include at least one of $t_{J}$ and $t_{K}$, and by Lemma 2] $\beta \geq 0$.

As all the coefficients in $\Lambda$ are non-negative and all the values of $t_{I}$ are nonnegative, its value is always non-negative. That is,

Lemma 4. Let $\sum_{I \subseteq[m]} \alpha_{I} H\left(X_{I}\right) \geq 0$ be an information inequality. Then, for every sets $A_{1}, \ldots, A_{m} \subseteq P, \sum_{I \subseteq[m]} \alpha_{I} C_{n}\left(\left|A_{I}\right|\right) \geq 0$.

\subsection{An Algorithm for Checking If an Information Inequality Cannot Help}

We next present the algorithm that checks if an information inequality cannot help. The algorithm is a brute-force algorithm that checks, for each possible choice of adding the dealer or not adding the dealer to each set $A_{i}$ and for each possible choice $A_{I} \in \mathcal{A}$ or $A_{I} \notin \mathcal{A}$ for each $I \subseteq[m]$, if $\Delta>0$ while it is possible that $\Lambda=0$. To check if $\Lambda$ can equal 0 under some a specific choice, we check for each choice $t_{I}=0$ and $t_{I}>0$ for each $I \subseteq[m]$ if (1) $\Lambda=0$ under this choice, and (2) this choice is consistent with the choice of sets that are in the access structure. The algorithm is formally described in Algorithm 11. 


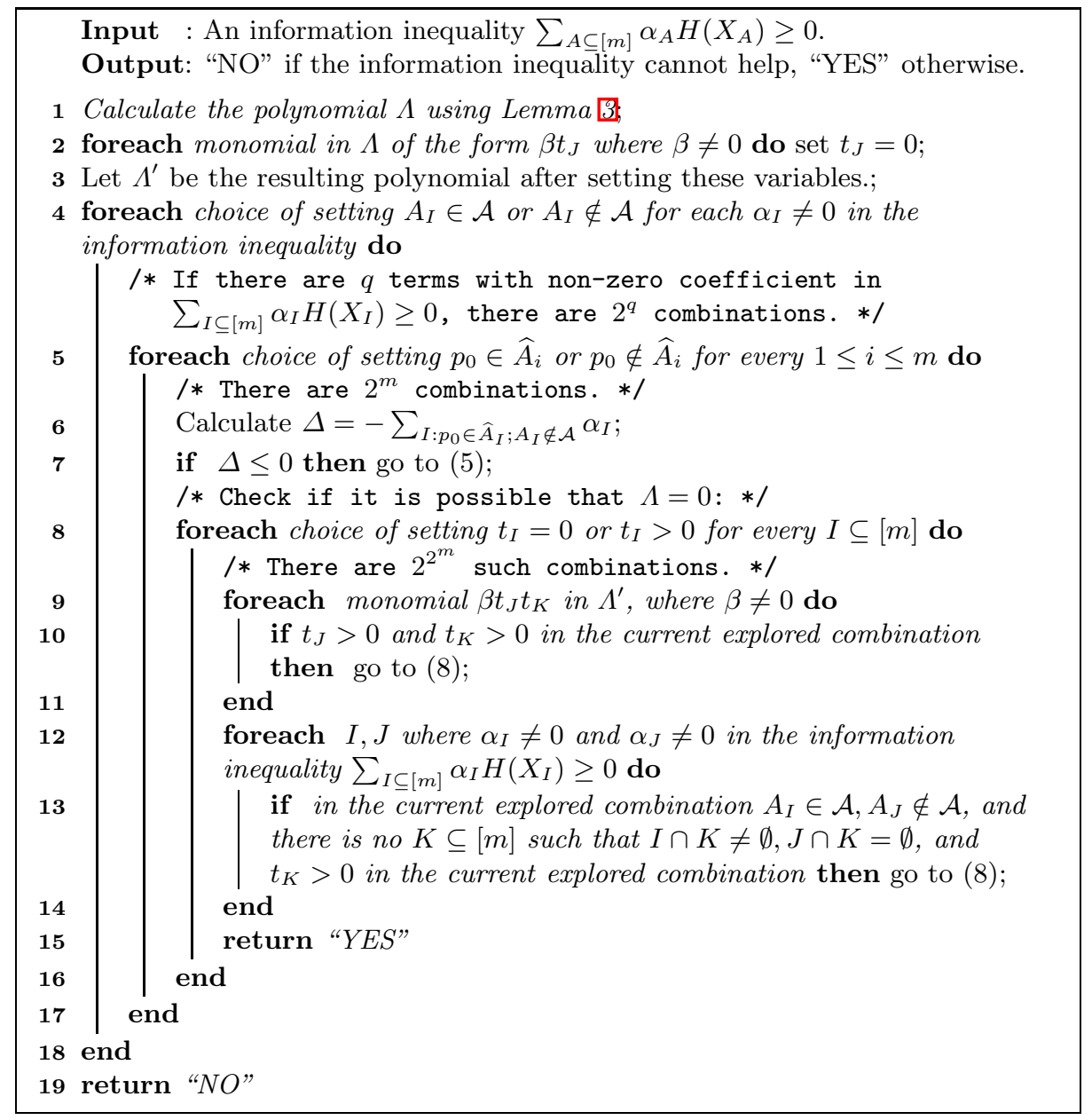

Algorithm 1. A brute-force algorithm that checks if an information inequality cannot help.

We have executed Algorithm 1 on the following non-Shannon inequalities:

- The first Non-Shannon inequality with four variables that was discovered by Zhang and Yeung in 47 .

- The six Non-Shannon inequalities with four variables and anther one with five variables in 23 .

- The five Non-Shannon inequalities with four variables in 44.

- The inequality of Ingleton in 29] 4

For each of these inequalities the result is the same - the information inequality cannot help in proving lower bounds of $\omega(n)$.

${ }^{4}$ The inequality of Ingleton [29] holds only for linear-algebraic spaces. 
Remark 2. The algorithm written above is not efficient. However, for our purpose - checking information inequalities with four or five variables - the algorithm is good enough. To be precise, the running of the computer program executing the algorithm takes less than a minute even for an information inequality of [23] that contains five variables. All other inequalities contain 4 variables and the running time is better.

Remark 3. Our algorithm gives a necessary condition for an information inequality to be helpful. We do not know of an information inequality that fulfills this necessary condition. We do have an example of a potential inequality that satisfies it: $H\left(X_{1} X_{2}\right)+H\left(X_{1} X_{3}\right)+H\left(X_{2} X_{3}\right)+H\left(X_{4}\right) \leq H\left(X_{1} X_{2} X_{3}\right)+H\left(X_{1}\right)+$ $H\left(X_{2}\right)+H\left(X_{3} X_{4}\right)$. We stress that we do not know if this is an information inequality. It does satisfy Lemma 2$]$ and some stronger conditions for being an information inequality.

\subsection{Dealing with the Known Infinite Collections of Information Inequalities}

There are a few examples for infinite sequences of Non-Shannon inequalities. The first infinite sequence of Non-Shannon inequalities was discovered by Zhang and Yeung in 47]; they show for every $n \in \mathbb{N}$ an information inequality with $n$ variables. A sequence of Non-Shannon information inequalities generalizing the result of [47] appears in [33, 46. Finally, an infinite sequence of Non-Shannon information inequalities with four variables was given in 44 .

In 44 there is a symbolic inequality with four variables, where some of the coefficients are a function of a parameter $s$. This inequality is an information inequality for every assignment $s \in \mathbb{N}^{+}$. For example, for $s=2$ it yields the Zhang and Yeung information inequality 47. For this symbolic information inequality, we computed the symbolic polynomial $\Lambda$ and proved that there is a constant $\gamma>0$ such that for every $s \in \mathbb{N}^{+}$the information inequality with parameter $s$ can at most $\gamma$-help. We used a similar technique to deal with the infinite sequence presented in [46] that is more general than the infinite sequences presented in [47, 33. For these sequences the result is that there is a constant $\gamma>0$ such that every inequality in the sequence can at most $\gamma$-help.

Using Theorem [2] we conclude that all the known information inequalities cannot help in proving lower bounds of $\omega(n)$ on the size of the shares in secretsharing schemes.

Theorem 4. The information inequalities of [29, 47, 33, 46, [23, 44] cannot help in proving lower bounds of $\omega(n)$ even if they are used simultaneously.

Acknowledgment. We thank the anonymous TCC referees for valuable comments.

\section{References}

[1] Babai, L., Gál, A., Wigderson, A.: Superpolynomial lower bounds for monotone span programs. Combinatorica 19(3), 301-319 (1999)

[2] Beimel, A., Chor, B.: Universally ideal secret sharing schemes. IEEE Trans. on Info. Theory 40(3), 786-794 (1994) 
[3] Beimel, A., Franklin, M.: Weakly-private secret sharing schemes. In: Vadhan, S.P. (ed.) TCC 2007. LNCS, vol. 4392, pp. 253-272. Springer, Heidelberg (2007)

[4] Beimel, A., Livne, N., Padró, C.: Matroids can be far from ideal secret sharing. In: Canetti, R. (ed.) TCC 2008. LNCS, vol. 4948, pp. 194-212. Springer, Heidelberg (2008)

[5] Bellare, M., Rogaway, P.: Robust computational secret sharing and a unified account of classical secret-sharing goals. In: 14th CCS, pp. 172-184 (2007)

[6] Ben-Or, M., Goldwasser, S., Wigderson, A.: Completeness theorems for noncryptographic fault-tolerant distributed computations. In: 20th STOC, pp. 1-10 (1988)

[7] Benaloh, J.C., Leichter, J.: Generalized secret sharing and monotone functions. In: Goldwasser, S. (ed.) CRYPTO 1988. LNCS, vol. 403, pp. 27-35. Springer, Heidelberg (1990)

[8] Blakley, G.R.: Safeguarding cryptographic keys. In: Proc. of the 1979 AFIPS National Computer Conference, pp. 313-317 (1979)

[9] Blundo, C., De Santis, A., Gargano, L., Vaccaro, U.: On the information rate of secret sharing schemes. Theoretical Computer Science 154(2), 283-306 (1996)

[10] Blundo, C., De Santis, A., Vaccaro, U.: On secret sharing schemes. Inform. Process. Lett. 65(1), 25-32 (1998)

[11] Brickell, E.F.: Some ideal secret sharing schemes. Journal of Combin. Math. and Combin. Comput. 6, 105-113 (1989)

[12] Capocelli, R.M., De Santis, A., Gargano, L., Vaccaro, U.: On the size of shares for secret sharing schemes. J. of Cryptology 6(3), 157-168 (1993)

[13] Chan, T.H., Yeung, R.W.: On a relation between information inequalities and group theory. IEEE Trans. on Info. Theory 48(7), 1992-1995 (2002)

[14] Chaum, D., Crépeau, C., Damgård, I.: Multiparty unconditionally secure protocols. In: 20th STOC, pp. 11-19 (1988)

[15] Chor, B., Kushilevitz, E.: Secret sharing over infinite domains. J. of Cryptology 6(2), 87-96 (1993)

[16] Cover, T.M., Thomas, J.A.: Elements of Information Theory. John Wiley \& Sons, Chichester (1991)

[17] Cramer, R., Damgård, I.B., Maurer, U.M.: General secure multi-party computation from any linear secret-sharing scheme. In: Preneel, B. (ed.) EUROCRYPT 2000. LNCS, vol. 1807, pp. 316-334. Springer, Heidelberg (2000)

[18] Csirmaz, L.: The size of a share must be large. In: De Santis, A. (ed.) EUROCRYPT 1994. LNCS, vol. 950, pp. 13-22. Springer, Heidelberg (1995)

[19] Csirmaz, L.: The dealer's random bits in perfect secret sharing schemes. Studia Sci. Math. Hungar. 32(3-4), 429-437 (1996)

[20] Desmedt, Y.G., Frankel, Y.: Shared generation of authenticators and signatures. In: Feigenbaum, J. (ed.) CRYPTO 1991. LNCS, vol. 576, pp. 457-469. Springer, Heidelberg (1992)

[21] van Dijk, M.: A linear construction of perfect secret sharing schemes. In: De Santis, A. (ed.) EUROCRYPT 1994. LNCS, vol. 950, pp. 23-34. Springer, Heidelberg (1995)

[22] van Dijk, M.: On the information rate of perfect secret sharing schemes. Designs, Codes and Cryptography 6, 143-169 (1995)

[23] Dougherty, R., Freiling, C., Zeger, K.: Six new non-Shannon information inequalities. In: ISIT 2006, pp. 233-236 (2006)

[24] Dougherty, R., Freiling, C., Zeger, K.: Networks, matroids, and non-Shannon information inequalities. IEEE Trans. on Info. Theory 53(6), 1949-1969 (2007)

[25] Fujishige, S.: Polymatroidal dependence structure of a set of random variables. Information and Control 39(1-3), 55-72 (1978) 
[26] Gál, A.: A characterization of span program size and improved lower bounds for monotone span programs. Computational Complexity 10(4), 277-296 (2002)

[27] Goyal, V., Pandey, O., Sahai, A., Waters, B.: Attribute-based encryption for finegrained access control of encrypted data. In: 13th CCS, pp. 89-98 (2006)

[28] Guille, L., Chan, T.H., Grant, A.: The minimal set of Ingleton inequalities. Technical Report 0802.2574, arxiv.org (2008), http://arxiv.org/abs/0802.2574

[29] Ingleton, A.W.: Conditions for representability and transversability of matroids. In: Proc. Fr. Br. Conf. 1970, pp. 62-67. Springer, Heidelberg (1971)

[30] Ito, M., Saito, A., Nishizeki, T.: Secret sharing schemes realizing general access structure. In: Globecom 1987, pp. 99-102 (1987)

[31] Karchmer, M., Wigderson, A.: On span programs. In: Proc. of the 8th IEEE Structure in Complexity Theory, pp. 102-111 (1993)

[32] Karnin, E.D., Greene, J.W., Hellman, M.E.: On secret sharing systems. IEEE Trans. on Info. Theory 29(1), 35-41 (1983)

[33] Makarychev, K., Makarychev, Y., Romashchenko, A., Vereshchagin, N.: A new class of non-Shannon type inequalities for entropies. Communications in Information and Systems 2(2), 147-166 (2002)

[34] Matúš, F.: Infinitely many information inequalities. In: IEEE International Symposium on Information Theory 2007, pp. 41-44 (2007)

[35] Matúš, F.: Two constructions on limits of entropy functions. IEEE Trans. on Info. Theory 53(1), 320-330 (2007)

[36] Naor, M., Wool, A.: Access control and signatures via quorum secret sharing. IEEE Transactions on Parallel and Distributed Systems 9(1), 909-922 (1998)

[37] Oxley, J.G.: Matroid Theory. Oxford University Press, Oxford (1992)

[38] Rabin, M.O.: Randomized Byzantine generals. In: Proc. of the 24th IEEE Symp. on Foundations of Computer Science, pp. 403-409 (1983)

[39] Riis, S.: Graph entropy, network coding and guessing games. Technical Report 0711.4175, arxiv.org (2007), http://arxiv.org/abs/0711.4175

[40] Shamir, A.: How to share a secret. Comm. of the ACM 22, 612-613 (1979)

[41] Shannon, C.E.: Communication theory of secrecy systems. Bell System Technical Journal 28(4), 656-715 (1949)

[42] Simmons, G.J., Jackson, W., Martin, K.M.: The geometry of shared secret schemes. Bulletin of the ICA 1, 71-88 (1991)

[43] Waters, B.: Ciphertext-policy attribute-based encryption: An expressive, efficient, and provably secure realization. Technical Report 2008/290, Cryptology ePrint Archive (2008), http://eprint.iacr.org/

[44] Xu, W., Wang, J., Sun, J.: A projection method for derivation of non-Shannontype information inequalities. In: ISIT 2008, pp. 2116-2120 (2008)

[45] Yeung, R.W.: A First Course in Information Theory. Springer, Heidelberg (2006)

[46] Zhang, Z.: On a new non-Shannon type information inequality. Communications in Information and Systems 3(1), 47-60 (2003)

[47] Zhang, Z., Yeung, R.W.: On characterization of entropy function via information inequalities. IEEE Trans. on Info. Theory 44(4), 1440-1452 (1998) 